\title{
Anatomical Configurations of Dominant Anastomotic Veins of the Superficial Cortical Venous System
}

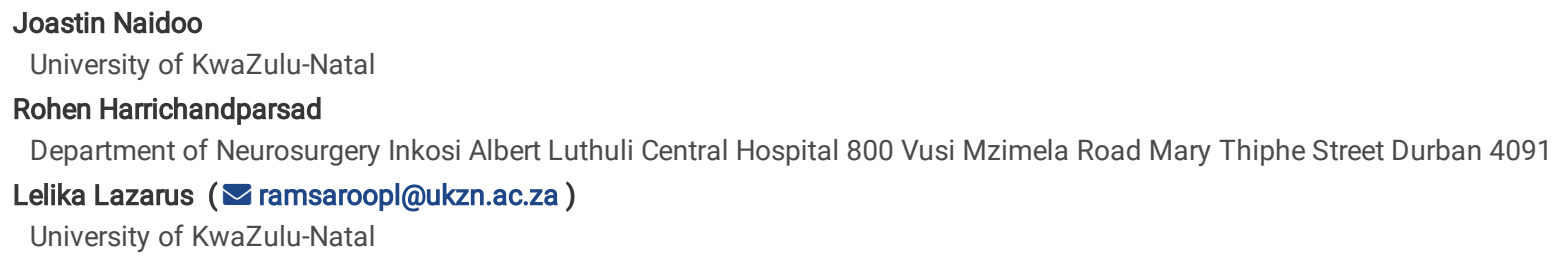




\section{Abstract}

Understanding the anatomy of the anastomotic veins (AV) of the superficial cortical venous system (SCVS), viz. superficial Sylvian vein (SSV) - also known as the superficial middle cerebral vein; vein of Labbe (VL) and vein of Trolard (VT), are imperative for neurosurgical procedures. This study aimed to investigate variant anatomical patterns of dominance of the $\mathrm{AV}$, to elucidate the haemodynamically balanced SCVS, by reporting variations between the presence, diameter and dominant patterns of the AV. Two hundred lateral angiograms were included, depicting left and right cerebral hemispheres of the same patient ( $n=100$ patients). Angiograms were analysed and variations recorded. Results were statistically compared against laterality, age, sex and ethnicity. Presence of the VL had the highest occurrence (96.5\%), whereas the SSV and VT had an occurrence of $75.5 \%$ and $64.5 \%$, respectively. This study reports presence of double veins of the AV: SSV (12.0\%), VL (22.0\%) and VT (19.5\%). Furthermore, presence of a triple vein for each AV is reported. Diameters for the SSV, VL and VT were $1.99 \pm 0.500 \mathrm{~mm}, 2.18 \pm 0.579 \mathrm{~mm}$ and $2.14 \pm 0.472 \mathrm{~mm}$, respectively. Statistically significant relationships were established between diameters and the SSV, VL, VT and VT2 (double VT). Seven types of dominant patterns were recorded: Equilibrium; singular dominance of SSV, VL and VT; co-dominance of SSV/VL, SSV/VT and VL/VT. The Equilibrium dominant pattern of drainage had the highest occurrence (54.5\%). Patterns of dominance of these $\mathrm{AV}$ can aid the neurosurgeon in curbing the risk of iatrogenic injury and postoperative infarcts even after an otherwise successful surgery.

\section{Introduction}

The superficial cortical venous system (SCVS) is a complex system consisting of dural venous sinuses and cortical veins ${ }^{1}$. Cortical veins are large subarachnoid venous collectors that receive venous drainage from a network of subpial veins, which lie beneath the arterial circulation of the cerebral cortex, and drains the superficial surface of the cerebrum ${ }^{2,3,4,5}$. These cortical veins are divided into three collecting groups and are linked via anastomotic veins (AV). These AV are large venous channels present in each of the collecting groups, and drain into their respective dural venous sinuses, viz. (i) the superficial middle cerebral vein of the anterior group, which drains into the cavernous or sphenoparietal sinus, (ii) inferior AV of the posteroinferior group, which drains into the transverse sinus and (iii) superior $\mathrm{AV}$ of the mediodorsal group, which drains into the superior sagittal sinus ${ }^{1,4,6}$. The $A V$, which provide an alternate pathway of drainage, are commonly referred to by eponyms; therefore, the superficial middle cerebral vein is termed as the superficial Sylvian vein (SSV), inferior AV as the vein of Labbe (VL), and superior AV as the vein of Trolard (VT) ${ }^{4,6,7,8}$.

The SSV originates in the posterior aspect of the lateral fissure, drains the region of the opercula and courses anteroinferiorly to drain into the cavernous sinus or sphenoparietal sinus ${ }^{4,9,10}$. The posterior end of the SSV anastomoses with the VL inferiorly and VT superiorly ${ }^{7,11}$. The largest anastomotic channel connecting the SSV and transverse sinus is the $\mathrm{VL}^{11,12}$. The $\mathrm{VL}$ originates in the posterior aspect of the lateral fissure and courses obliquely over the occipitotemporal sulcus, as well as the inferolateral aspect of the temporal lobe, to project posteroinferiorly into the transverse sinus ${ }^{9,12}$. The VT is the largest vein that crosses the lateral surface of the frontal or parietal lobes between the SSV and superior sagittal sinus ${ }^{5,8}$. VT has the most variable course, and while reported to be located in the area of the central sulcus, this AV may be present as anteriorly as the anterior frontal vein or as posteriorly as the anterior parietal vein ${ }^{4,8,9}$. Due to the presence of these AV, the SCVS is haemodynamically balanced since the SSV anastomoses with the VL inferiorly and VT superiorly 4,7 .

Regarding presence of the $A V$, the literature reports the presence of double $A V$ of the SSV, VL and VT, respectively $4,5,8,13,14,15$. While the SCVS is known to be a haemodynamically balanced system, in which drainage of the cortical hemispheres into the venous sinuses was noted as being equally distributed, the anatomical structure of the veins of the SCVS may differ ${ }^{7,8}$. The AV may range from interconnected or separated $A V$, to a single dominant $A V$ while the other two AV are hypoplastic ${ }^{4}$. Furthermore, Tanriverdi et al. reported variations of combinations of $A V$ as the dominant drainage pattern for the SCVS, in which two of the three AV are co-dominant ${ }^{8}$. Therefore, although the SSV, VL and VT have been noted to have equal sizes, it is not uncommon for one or two of these AV to predominate and the others to be hypoplastic ${ }^{4,14}$.

The variant patterns of anastomosis of these AV have important surgical implications ${ }^{16}$. In well-developed anastomoses of the AV, particular cortical veins could be safely sacrificed; however, in cases of poor collaterals, preservation of the AV is essential'. AV are regarded as 'dangerous veins' since the risk of iatrogenic injury would result in severe complications such as oedema, swelling, raised intracranial pressure and haemorrhagic infarcts ${ }^{16,17}$. Neglecting to recognise an SCVS in which a singular AV is dominant may result in a venous infarct, even after an otherwise successful surgical procedure, due to the drainage route being compromised ${ }^{4,5,8}$. Since the $A V$ are generally encountered during pterional craniotomies, which is a common neurosurgical approach, the risk of iatrogenic injury to the $\mathrm{AV}$ must be reduced; therefore, the anatomy regarding the $\mathrm{AV}$ in terms of their presence and dominance becomes imperative for neurosurgical perspectives ${ }^{4,8,12,18}$

However, while cortical veins contain $70 \%$ of the cerebral blood volume, few studies have focused on the venous drainage of the cerebral cortex in comparison to the arterial supply ${ }^{9}$. Knowledge of the variations of the AV of the SCVS would then elucidate the system ${ }^{9}$. Therefore, this study aimed to investigate the anatomical patterns of dominance of the AV of the SCVS. The objectives were to document the presence of the AV, determine the diameter, as well as record patterns of dominance and equidominance, and compare these factors on the basis of laterality as well as age, sex and ethnicity.

\section{Materials And Methods}

\section{Patient cohort}


A retrospective chart review of 200 lateral angiograms ( $n=100$ left; 100 right), were used. Therefore, a cohort of 100 patients was included in this study; demographics were recorded as depicted in Table 1. Angiograms were obtained from the Department of Neurosurgery, Inkosi Albert Luthuli Central Hospital, Durban, South Africa. Relevant Gatekeeper permissions were sough. The Biomedical Research Ethics Committee of the University of KwaZulu-Natal approved the study (BREC/00001676/2020) and waived the need for informed consent as this study utilized retrospective chart reviews. There was no patient contact, and no patient details were released from images. All methods were carried out in accordance with relevant guidelines and regulations.

Table 1

Demographics of patient cohort

\begin{tabular}{|c|c|}
\hline & Patients \\
\hline Total & 100 \\
\hline \multicolumn{2}{|c|}{ LATERALITY } \\
\hline Left & 100 \\
\hline Right & 100 \\
\hline \multicolumn{2}{|c|}{ AGE (Years) } \\
\hline$<20$ & 15 \\
\hline $20-29$ & 16 \\
\hline $30-39$ & 20 \\
\hline $40-49$ & 22 \\
\hline $50-59$ & 13 \\
\hline$\geq 60$ & 14 \\
\hline \multicolumn{2}{|l|}{ SEX } \\
\hline Female & 60 \\
\hline Male & 40 \\
\hline \multicolumn{2}{|c|}{ ETHNICITY } \\
\hline African & 74 \\
\hline Coloured & 2 \\
\hline Indian & 15 \\
\hline White & 09 \\
\hline
\end{tabular}

\section{Data processing}

Angiograms were observed and analysed by use of the RadiAnt DICOM Viewer software. Angiograms with pathology affecting the venous anatomy, e.g arteriovenous malformations (AVMs) and dural arteriovenous fistulae (DAVF), were excluded. Only angiograms depicting both cerebral hemispheres of the same patient were included.

\section{Data collection}

In the event of a double or multiple occurrences of an $\mathrm{AV}$, the respective veins were numbered sequentially in an anterior to posterior (VL and $\mathrm{VT}$ ) or superior to inferior (SSV) direction, e.g. SSV, SSV2, SSV3, of which SSV is the most anterosuperior of the three veins and SSV3 the most posteroinferior.

Diameter of the vein was documented at the widest observable point of the vessel, as well as $5.00 \mathrm{~mm}$ proximal and distal to the said point. All measurements were taken three times to ensure reliability, interclass correlation coefficients were calculated to determine the reliability of the measurements (confidence intervals: 95\%). An average of these measurements was then used for statistical analysis.

Dominance of the AV was ascertained by the territory of which the AV drained. A dominant AV was determined if one of the AV, viz. SSV, VL or VT, was visualised as being the main venous drainage of the territory of another AV. A haemodynamically balanced SCVS with an equilibrium dominance pattern was determined if all AV presents drain their respective territories. These dominant patterns were then recorded.

\section{Statistical analysis}

Statistical analysis was performed by using R statistical computing software, version 3.63 (R Studio, Boston, MA, USA). A statistically significant value of $p$ $<0.05$ was set. The Chi-square and Fisher's exact tests were employed for association between categorical variables. The t-test and Rank-sum test were employed to determine the ( \pm standard deviation) and median (inter-quartile) differences of numerical variables between categorical variables for normally and non-normally distributed data, respectively, when comparing two groups. Whereas the Kruskal-Wallis test and ANOVA F- test was used to determine mean ( \pm standard deviation) and median (inter-quartile) differences of numerical variables between categorical variables for normally and non-normally 
distributed data, respectively, for comparing more than two groups. A Cramers V correlation was performed to determine a relationship between the dominant patterns and laterality.

\section{Results}

The following results were obtained after analysis of the lateral angiograms included in the retrospective chart review.

Results of an intra-rater reliability test infer that the average measurements used are highly reliable $(\mathrm{p}<0.001)$, interclass coefficients for all intra-rater reliability tests were $(I C C=1)$. The inter-rater reliability tests yielded the following interclass coefficients (confidence interval: $95 \%)$ : SSV Left $(I C C=1)$, SSV2 Left (ICC=1), VL Left (ICC=1), VT Left (ICC=0.96), VT2 Left (ICC=0.99), SSV Right (ICC=1), VL Right (ICC=0.98), VL2 Right (ICC=0.85), VT Right (ICC=0.99).

\section{Table 2}

Presence of the anastomotic veins

\begin{tabular}{|c|c|c|c|c|}
\hline & \multirow[t]{2}{*}{ Hemispheres } & \multicolumn{3}{|c|}{ PRESENCE [n (\%)] } \\
\hline & & ssv & VL & VT \\
\hline Total & 200 & $151(75.5)$ & $193(96.5)$ & $129(64.5)$ \\
\hline \multicolumn{5}{|c|}{ LATERALITY } \\
\hline Left & 100 & $79(79.0)$ & $97(97.0)$ & $61(61.0)$ \\
\hline Right & 100 & $72(72.0)$ & $96(96.0)$ & $68(68.0)$ \\
\hline$p$ value & & $0.250^{a}$ & $1.000^{b}$ & $0.301^{a}$ \\
\hline \multicolumn{5}{|c|}{ AGE (Years) } \\
\hline$<20$ & 30 & $23(76.7)$ & $28(93.3)$ & $18(60.0)$ \\
\hline $20-29$ & 32 & $19(59.4)$ & $32(100)$ & $23(71.9)$ \\
\hline $30-39$ & 40 & $28(70.0)$ & $39(97.5)$ & $19(47.5)$ \\
\hline $40-49$ & 44 & $37(84.1)$ & $42(95.5)$ & $28(63.6)$ \\
\hline $50-59$ & 26 & $21(80.8)$ & $25(96.2)$ & $18(69.2)$ \\
\hline$\geq 60$ & 28 & $23(82.1)$ & $27(96.4)$ & $23(82.1)$ \\
\hline$p$ value & & $0.157^{a}$ & $0.829^{b}$ & $0.071^{a}$ \\
\hline \multicolumn{5}{|l|}{ SEX } \\
\hline Female & 120 & $93(77.5)$ & $115(95.8)$ & $72(60.0)$ \\
\hline Male & 80 & $58(72.5)$ & $78(97.5)$ & 57 (71.2) \\
\hline$p$ value & & $0.421^{a}$ & $0.704^{b}$ & $0.103^{a}$ \\
\hline \multicolumn{5}{|c|}{ ETHNICITY } \\
\hline African & 148 & $111(75.0)$ & $142(95.9)$ & $93(62.8)$ \\
\hline Coloured & 4 & $3(75.0)$ & $4(100)$ & $3(75.0)$ \\
\hline Indian & 30 & $21(70.0)$ & $30(100)$ & $19(63.3)$ \\
\hline White & 18 & $16(88.9)$ & $17(94.4)$ & $14(77.8)$ \\
\hline$p$ value & & $0.494^{b}$ & $0.474^{b}$ & $0.672^{b}$ \\
\hline \multicolumn{5}{|l|}{ KEY: } \\
\hline \multirow{2}{*}{\multicolumn{5}{|c|}{ SSV - superficial Sylvian vein, VL - vein of Labbe, VT - vein of Trolard }} \\
\hline${ }^{a}$ Chi-squar & test ${ }^{b}$ Fisher's & & & \\
\hline
\end{tabular}

We recorded presence of the three AV, viz. SSV, VL and VT in $75.5 \%, 96.5 \%$ and $64.5 \%$ of the sample, respectively. VT had a higher occurrence in the right hemisphere (68.0\%) than the left (61.0\%), whereas the left hemisphere had a higher occurrence of VL (97.0\%) in comparison to the right hemisphere (96.0\%). Patients in the age group of 20 - 29 years had a 100\% occurrence of the VL; however, this group also presented with the lowest occurrence of the SSV (59.4\%). Males had higher occurrences of the VL (97.5\%) and VT (71.2\%), yet a lower occurrence of the SSV (72.5\%). The Indian ethnic group had the lowest 
occurrence of SSV (70.0\%), whereas the African ethnic group presented with the lowest occurrence of the VT (62.8\%). Presence of the AV, among the demographics, was not found to be statistically significant $(p>0.05)$ (Table 2, Fig.1).

This study also recorded the presence of double veins: Double SSV - 12.0\% (left: 13.0\%; right: 11.0\%), Double VL - 22.0\% (left: 19.0\%; right: 25.0\%), Double VT - 19.5\% (left: 15.0\%; right: 24.0\%) (Fig.1).

Furthermore, we report findings of triple presence of each AV. A triple SSV was present in the right hemisphere of a 51-year-old Indian female. The triple VL was also recorded in the left hemisphere of a 16-year-old African female and 45-year-old White female. Triple VT occurred in the right hemisphere of a 25year-old African male (Fig.1).

Table 3

Mean diameters of the anastomotic veins

\begin{tabular}{|c|c|c|c|c|c|c|c|c|c|c|}
\hline & \multirow[t]{2}{*}{ Hemispheres } & \multicolumn{9}{|c|}{ DIAMETER $(\mathrm{mm})$} \\
\hline & & ssV & SSV2 & SSV3 & VL & VL2 & VL3 & VT & VT2 & VT3 \\
\hline Total & 200 & $1.99 \pm 0.500$ & $1.92 \pm 0.477$ & 2.68 & $2.18 \pm 0.579$ & $1.96 \pm 0.388$ & $1.52 \pm 0.0424$ & $2.14 \pm 0.472$ & $2.19 \pm 0.604$ & 1.63 \\
\hline \multicolumn{11}{|c|}{ LATERALITY } \\
\hline Left & 100 & $1.90 \pm 0.470$ & $1.91 \pm 0.513$ & & $2.14 \pm 0.598$ & $1.85 \pm 0.318$ & 1.49 & $2.14 \pm 0.488$ & $2.23 \pm 0.538$ & \\
\hline Right & 100 & $2.08 \pm 0.516$ & $1.94 \pm 0.462$ & 2.68 & $2.21 \pm 0.560$ & $2.03 \pm 0.419$ & 1.55 & $2.14 \pm 0.460$ & $2.15 \pm 0.660$ & 1.63 \\
\hline$p$ value & & $0.024^{* a}$ & $0.856^{b}$ & - & $0.285^{a}$ & $0.119^{b}$ & - & $0.998^{b}$ & $0.706^{b}$ & - \\
\hline \multicolumn{11}{|c|}{ AGE (Years) } \\
\hline$<20$ & 30 & $2.07 \pm 0.454$ & $2.15 \pm 0.694$ & & $2.15 \pm 0.594$ & $2.06 \pm 0.456$ & 1.49 & $2.02 \pm 0.450$ & $2.57 \pm 0.340$ & \\
\hline $20-29$ & 32 & $2.15 \pm 0.680$ & $1.95 \pm 0.368$ & & $2.27 \pm 0.675$ & $2.04 \pm 0.243$ & & $2.07 \pm 0.397$ & $1.84 \pm 0.381$ & 1.63 \\
\hline $30-39$ & 40 & $2.02 \pm 0.499$ & $1.83 \pm 0.066$ & & $2.39 \pm 0.504$ & $1.87 \pm 0.504$ & & $2.39 \pm 0.509$ & $2.50 \pm 0.461$ & \\
\hline $40-49$ & 44 & $2.16 \pm 0.393$ & $1.74 \pm 0.438$ & & $2.04 \pm 0.527$ & $1.95 \pm 0.362$ & 1.55 & $2.31 \pm 0.483$ & $2.65 \pm 0.744$ & \\
\hline $50-59$ & 26 & $1.65 \pm 0.363$ & $1.56 \pm 0.442$ & 2.68 & $2.20 \pm 0.567$ & $1.79 \pm 0.318$ & & $1.98 \pm 0.423$ & $1.82 \pm 0.385$ & \\
\hline$\geq 60$ & 28 & $1.75 \pm 0.435$ & $2.06 \pm 0.195$ & & $1.98 \pm 0.552$ & $1.80 \pm 0.386$ & & $2.03 \pm 0.455$ & $1.68 \pm 0.261$ & \\
\hline$p$ value & & $<0.001^{* c}$ & $0.548^{d}$ & - & $0.025^{\star} c$ & - & - & $0.015^{\star d}$ & $0.003^{\star d}$ & - \\
\hline \multicolumn{11}{|l|}{ SEX } \\
\hline Female & 120 & $1.95 \pm 0.426$ & $1.92 \pm 0.570$ & 2.68 & $2.06 \pm 0.542$ & $1.97 \pm 0.436$ & $1.52 \pm 0.0424$ & $2.07 \pm 0.436$ & $2.08 \pm 0.629$ & \\
\hline Male & 80 & $2.04 \pm 0.598$ & $1.93 \pm 0.194$ & $2.35 \pm 0.590$ & $1.95 \pm 0.309$ & $2.23 \pm 0.504$ & $2.30 \pm 0.574$ & 1.63 & & \\
\hline$p$ value & & $0.477^{a}$ & $0.907^{a}$ & - & $<0.001^{\star b}$ & $0.906^{b}$ & - & $0.062^{b}$ & $0.031^{b}$ & - \\
\hline \multicolumn{11}{|c|}{ ETHNICITY } \\
\hline African & 148 & $1.97 \pm 0.508$ & $1.95 \pm 0.495$ & & $2.21 \pm 0.599$ & $2.00 \pm 0.387$ & 1.49 & $2.12 \pm 0.456$ & $2.07 \pm 0.534$ & 1.63 \\
\hline Coloured & 4 & $2.01 \pm 0.355$ & & & $1.69 \pm 0.724$ & 2.24 & & $1.83 \pm 0.219$ & & \\
\hline Indian & 30 & $2.02 \pm 0.456$ & $1.68 \pm 0.537$ & 2.68 & $2.25 \pm 0.470$ & $1.91 \pm 0.381$ & & $2.20 \pm 0.602$ & $2.62 \pm 0.778$ & \\
\hline White & 18 & $2.07 \pm 0.546$ & $1.86 \pm 0.354$ & & $1.91 \pm 0.453$ & $1.67 \pm 0.371$ & 1.55 & $2.30 \pm 0.394$ & $2.10 \pm 0.451$ & \\
\hline$p$ value & & $0.799^{c}$ & - & - & $0.080^{c}$ & - & - & $0.342^{c}$ & - & - \\
\hline \multicolumn{11}{|l|}{ KEY: } \\
\hline \multicolumn{11}{|c|}{ SSV, SSV2, SSV3 - superficial Sylvian veins; single, double and triple occurrence, respectively. } \\
\hline \multicolumn{11}{|c|}{ VL, VL2, VL3 - vein of Labbe; single, double and triple occurrence, respectively. } \\
\hline \multicolumn{11}{|c|}{ VT, VT2, VT3 - vein of Trolard; single, double and triple occurrence, respectively. } \\
\hline \multicolumn{11}{|c|}{${ }^{a}$ Rank-sum test $\quad b_{t-T e s t}$} \\
\hline \multicolumn{8}{|c|}{${ }^{c}$ Krusker-Wallis test dANOVA F-Test } & & & \\
\hline *statistica & ly significant sin & ce $p<0.05$ & & & & & & & & \\
\hline
\end{tabular}


The mean diameter recorded for the SSV was $1.99 \pm 0.500 \mathrm{~mm}$. The SSV2 had a mean diameter of $1.92 \pm 0.477 \mathrm{~mm}$. A mean diameter of $2.18 \pm 0.579 \mathrm{~mm}$ was recorded for the VL, whereas VL2 had a mean diameter of $1.96 \pm 0.388 \mathrm{~mm}$. The recorded mean diameter for VT $(2.14 \pm 0.472 \mathrm{~mm})$ was smaller than that of the more posterior VT2 $(2.19 \pm 0.604 \mathrm{~mm})$. The diameters of SSV3, VL3 and VT3 were $2.68 \mathrm{~mm}, 1.52 \pm 0.0424 \mathrm{~mm}$ and $1.63 \mathrm{~mm}$, respectively (Table 3$)$.

A statistically significant relationship was reported between the SSV in left $(1.90 \pm 0.470 \mathrm{~mm})$ and right $(2.08 \pm 0.516 \mathrm{~mm})$ cerebral hemispheres, respectively $(p=0.024)$. Furthermore, the SSV had a strong, statistically significant relationship between the age groups $(p<0.001)$. Patients in the age group of $40-49$ years reported the largest mean diameter of SSV $(2.16 \pm 0.393 \mathrm{~mm})$, while patients in the 50 - 59 years and $\geq 60$ years age groups had a much smaller diameter of SSV with $1.65 \pm 0.363 \mathrm{~mm}$ and $1.75 \pm 0.435 \mathrm{~mm}$, respectively. Statistically significant relationships were also reported for the VL ( $\mathrm{p}=0.025$ ), VT $(p=0.015)$ and VT2 ( $p=0.003)$ among the age groups. A strong, statistically significant relationship was also determined between the mean diameter of VL in females $(2.06 \pm 0.542 \mathrm{~mm})$ and males $(2.35 \pm 0.590 \mathrm{~mm})(\mathrm{p}<0.001)$. However, females have a larger diameter of VL2 than males with $1.97 \pm 0.436 \mathrm{~mm}$ and $1.95 \pm 0.309$, respectively. The mean diameter of SSV $(2.07 \pm 0.546 \mathrm{~mm})$ and VT $(2.30 \pm 0.394 \mathrm{~mm})$ was largest amongst the White ethnic group; however, the VL was noted as being largest amongst the Indian ethnic group $(2.25 \pm 0.470 \mathrm{~mm})$ (Table 3$)$.

\section{Table 4}

Dominant patterns of the anastomotic veins

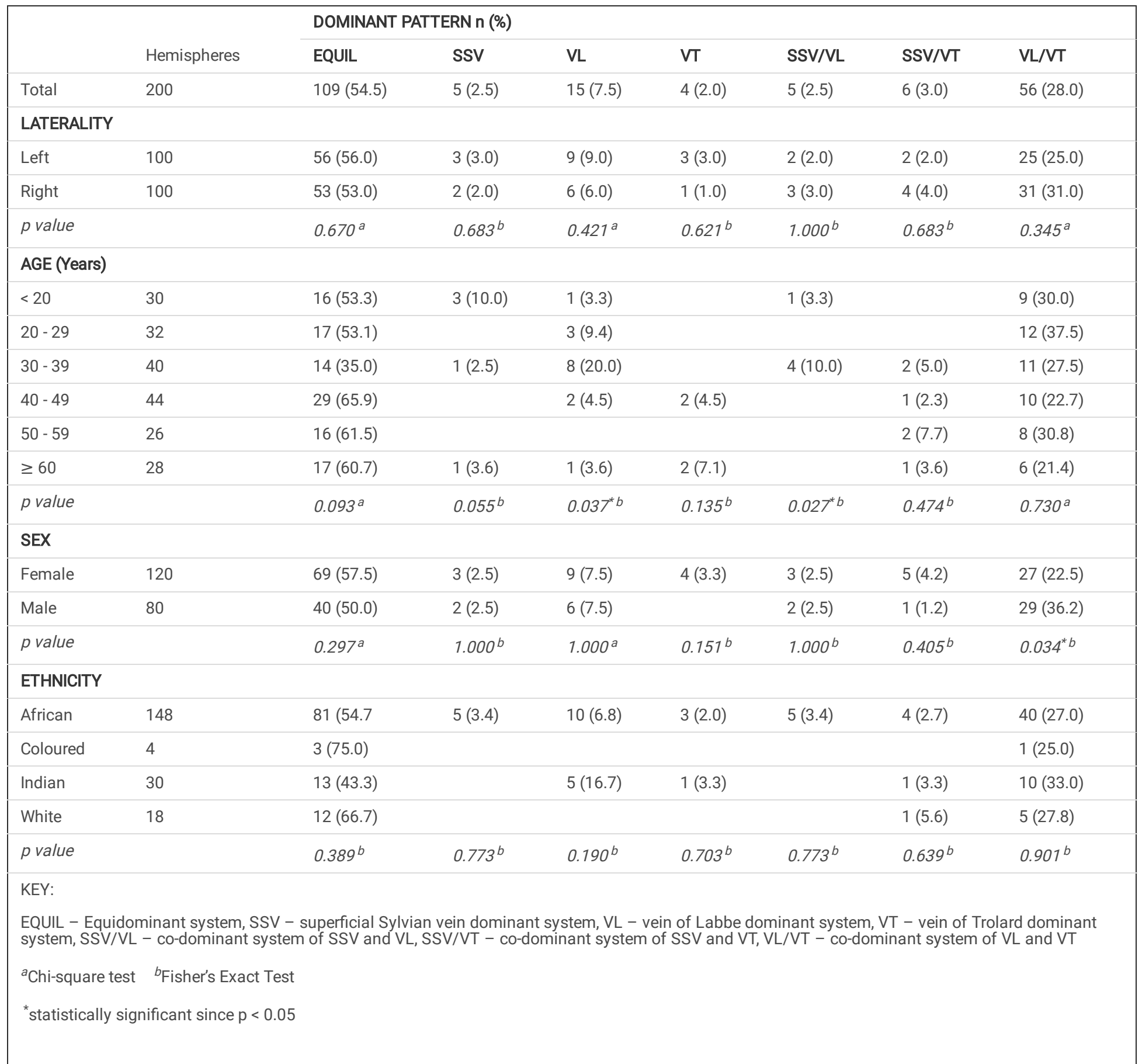


We report seven types of dominant patterns of the AV of the SCVS (Table 4, Fig.2). The pattern with the highest incidence is the Equilibrium pattern (54.5\%), followed by the VL/VT pattern (28.0\%).

The Equilibrium pattern had a higher occurrence on the left hemisphere (56.0\%) in comparison to the right hemisphere (53.0\%), whereas the VL/VT pattern had a higher occurrence on the right hemisphere when compared to the left with $31.0 \%$ and $25.0 \%$, respectively. A statistically significant relationship was determined with SSV/VL drainage patterns amongst the age groups $(p=0.027)$. SSV/VL occurrence in age groups of $<20$ years and $30-39$ years were $3.3 \%$ and $10.0 \%$, respectively. The VL dominant pattern also expressed a statistically significant relationship amongst the age groups ( $p=0.037)$, the age group of 30 - 39 years having a $20.0 \%$ occurrence of this type of drainage pattern; furthermore, this age group had the lowest occurrence of the Equilibrium type (35.0\%). The statistical relationship between the presence of VL/VT dominant pattern amongst males and females was found to be significant ( $p=0.034)$. Males had a higher occurrence of the VL/VT type than females with $36.2 \%$ and $22.5 \%$, respectively. The Indian ethnic group had the lowest occurrence of the Equilibrium dominant type (43.3\%) and the highest occurrence of the VL/VT dominant drainage type (33.0\%) (Table 4, Fig.2).

Table 5

\section{Cramers $V$ correlation of dominant patterns of the anastomotic veins based on laterality}

\begin{tabular}{|llllllll|}
\hline Correlation & & & & & & & \\
\hline EQUILIBRIUM LEFT & 0.22 & 0.02 & 0.20 & 0.09 & 0.06 & 0.02 & 0.10 \\
\hline SSV LEFT & 0.19 & 0.39 & 0.04 & 0.02 & 0.03 & 0.26 & 0.01 \\
\hline VL LEFT & 0.33 & 0.04 & 0.51 & 0.03 & 0.35 & 0.06 & 0.04 \\
\hline VT LEFT & 0.05 & 0.03 & 0.04 & 0.02 & 0.03 & 0.03 & 0.06 \\
\hline SSV/VL LEFT & 0.01 & 0.02 & 0.04 & 0.01 & 0.02 & 0.03 & 0.10 \\
\hline SSV/VT LEFT & 0.01 & 0.02 & 0.26 & 0.01 & 0.02 & 0.10 & 0.11 \\
\hline VL/VT LEFT & 0.04 & 0.06 & 0.15 & 0.06 & 0.10 & SSV/VT RIGHT VL/VT RIGHT \\
\hline TYPE & EQUILIBIRUM RIGHT & SSV RIGHT & VL RIGHT & VT RIGHT & SSV/VL RIGHT & S \\
\hline
\end{tabular}

According to Bergsma, a Cramers $V$ in the range of $[0,0.3]$ is considered weak, $[0.3,0.7]$ is considered medium and $>0.7$ as strong ${ }^{19}$. We report medium correlations between dominant drainage patterns of SSV Left and SSV Right (0.39), VL Left and Equilibrium Right (0.33), VL Left and VL Right (0.51) as well as VL Left and SSV/VL Right (0.35). There are no strong correlations reported between these dominant patterns based on laterality in the present study (Table 5).

\section{Discussion}

The anatomy of the veins of the SCVS is of interest to clinicians. Special focus lies in the anatomy of the AV, since these veins are regarded as 'dangerous veins' in the neurosurgical field, due to the high risk of postoperative infarction after an otherwise successful surgery ${ }^{7}$. However, there is a significant paucity of literature focusing on the AV, especially in the context of the SCVS entirely. This study aimed to fill the gap in the literature regarding the anatomy and anatomical variations of the AV to assist clinicians in preoperative planning. Therefore, familiarity with the variant anatomy of these AV will elucidate the SCVS since precise information regarding these veins would be able to form a network of reference points that are relevant during surgical planning and surgical procedures ${ }^{9,17}$.

\section{Presence}

Krings et al. stated that the VL or VT may be absent, but that the SSV would be invariably present ${ }^{4}$. We reported a $75.5 \%$ presence of the SSV in the present study. The SSV had a presence of $79.0 \%$ and $72.0 \%$ on the left and right cerebral hemispheres, respectively (Table 2). The occurrence of the SSV in the present study is lower than that reported by Kawamata et al. who reported a $99.2 \%$ presence in the left hemisphere and $95.1 \%$ in the right cerebral hemisphere ${ }^{20}$. Wangaryattawanich et al. stated that the SSV may be absent in $9.0-10.0 \%$ of individuals; this statement is corroborated by the study conducted by Kazumata et al., who reported an absent or hypoplastic SSV in $10.0 \%$ of the sample ${ }^{12,21}$. In the absence of the SSV, the territory is drained by the deep system of cortical veins, or the adjacent $\mathrm{AV}^{12,22}$. The SSV was reported to have a higher percentage of presence in females (77.5\%) than males (72.5\%), as well as a much higher presence in White ethnic group (88.9\%) when compared to the Indian ethnic group (70.0\%) (Table 2).

The VL had the highest occurrence, with a $96.5 \%$ presence in the total sample size. We also report $97.0 \%$ and $96.0 \%$ presence of the VL in the left and right cerebral hemispheres, respectively (Table 2). These results are much higher than those reported by Kawamata et al., who reported $64.8 \%$ in the left hemisphere and $41.8 \%$ in the right hemisphere ${ }^{20}$. The VL was determined to have a $100 \%$ occurrence in the $20-29$ years age group, as well as the Coloured and Indian ethnic groups (Table 2). The high occurrence of the VL must be noted by clinicians since a sacrifice of this vein in a poorly anastomosed SCVS would lead to a swollen temporal lobe and brainstem compression, as well as posterior haemorrhagic infarct ${ }^{5}$. 
We report a presence of the VT in $64.5 \%$ of the total sample size, with $61.0 \%$ and $68.0 \%$ presence in the left and right cerebral hemispheres, respectively (Table 2). These results are higher than those reported by Kawamata et al., with $59.0 \%$ in the left hemisphere and $54.9 \%$ in the right hemisphere ${ }^{20}$. In the absence of the VT, blood is drained by the superior cortical veins into the superior sagittal sinus or drained by an adjacent AV. Kilic and Akakin stated that on lateral angiograms, 8-12 superior cortical veins can be visualised, while Appaji et al. stated that there are 10-15 superior cortical veins; however, in the present study, a minimum of three and maximum of seven superior cortical veins draining the superolateral cerebral convexity in the absence of the VT was identified $^{11,18}$. This study reports a low occurrence of the presence of VT in the 30-39 year age group (47.5\%), a higher presence of VT in males (71.2\%) in comparison to females (60.0\%), and the lowest occurrence in the African ethnic group (62.8\%) (Table 2).

Each of the three AV have been reported to have double veins present, in a study conducted by Tanriverdi et al. it was reported that $9.9 \%$ of patients had a duplication of any of the three $\mathrm{AV}^{8}$. A vein was considered as duplicate if the $\mathrm{AV}$ had more than one distinct vein that drained the respective territory of the AV into its' respective dural venous sinuses, as seen in Figs. 1C-1F. We report a presence of $12 \%$ of double SSV (left: $13.0 \%$; right: $11.0 \%$ ); this is lower than the results of the study conducted by Bisaria, who reported a double bilateral SSV in $37.5 \%$ of their sample ${ }^{10}$. Double VL was also recorded in the present study, with an occurrence of $22.0 \%$ (left: $19.0 \%$; right: $25.0 \%$ ); this is corroborated by the studies conducted by Koperna et al. and Avci et al. who reported presence of double VL in $18.0 \%$ and $20.0 \%$ of their samples, respectively ${ }^{15,23}$. We also report a double VT in $19.5 \%$ (left: $15.0 \%$; right: $24.0 \%$ ) of the cases in this study.

Furthermore, this study includes the presence of triple AV, as seen in Figs. 1G-1L. The triple SSV and triple VT were present in right cerebral hemispheres, whereas both occurrences of the triple VL were present in left cerebral hemispheres. The triple VT was present in a male patient, while the triple SSV and triple VL were present in female patients. Although no statistically significant relationships were determined between sex and presence of the AV ( $p>0.05$ ), since the triple $A V$ is a novel finding this would be important to note. Knowledge of the triple AV variant is imperative for neurosurgical perspectives since this could affect the consequence of sacrifice of some of these AV and must be considered during preoperative planning.

\section{Diameter}

Sindou et al. stated that any of the veins of the SCVS with a certain calibre can be presumed to have a functional role ${ }^{16}$. We reported mean diameters for the SSV $(1.99 \pm 0.500 \mathrm{~mm}), \operatorname{SSV} 2(1.92 \pm 0.477 \mathrm{~mm}), \operatorname{SSV} 3(2.68 \mathrm{~mm}), \mathrm{VL}(2.18 \pm 0.579 \mathrm{~mm}), \mathrm{VL} 2(1.96 \pm 0.388 \mathrm{~mm}), \mathrm{VL} 3(1.52 \pm 0.0424 \mathrm{~mm}), \mathrm{VT}(2.14 \pm$ $0.472 \mathrm{~mm})$, VT2 $(2.19 \pm 0.604 \mathrm{~mm})$ and VT3 $(1.63 \mathrm{~mm})$ (Table 3).

Kawamata et al. classified the calibre of the AV into three groups based on their diameter: (i) small: $<1.00 \mathrm{~mm}$, (ii) medium: 1.00-3.00mm and (iii) large: > $3.00 \mathrm{~mm}^{20}$. The results of the present study indicate that the mean diameters fall within the medium calibre group. Silva et al. reported mean diameters of $3.2 \mathrm{~mm}$ and $3.32 \mathrm{~mm}$ for the VL and VT, respectively; these diameters are greater than those reported in the present study and could be attributed to the difference in population since Silva et al. studied the AV in a Portuguese cohort ${ }^{13}$. However, the results of the present study do not report any statistically significant results between the different ethnic groups within the South African cohort. Statistically significant results were reported among age groups, for the SSV $(p<0.001), V L(p=0.025)$, VT $(p=0.015)$ and VT2 $(p=0.003)$ (Table 3$)$. This relationship should be noted by clinicians since the $50-59$ year and $\geq$ 60 years age groups had relatively smaller diameters of the AV. A statistically significant relationship was also determined between females and males for the diameter of $\mathrm{VL}(\mathrm{p}<0.001)$, with the mean diameter of the $\mathrm{VL}$ in females $(2.06 \pm 0.542 \mathrm{~mm})$ being lower than that of males $(2.35 \pm 0.590 \mathrm{~mm})(T a b l e 3)$. Avci et al. stated that double VL were of equal size, whereas Krings et al. stated that if a double VL is present, the posterior VL tends to be larger ${ }^{4,23}$. The present study disagrees with both statements since the mean diameters of VL, VL2 and VL3 are vastly different; furthermore, the posterior VL, in this case VL2 and VL3, have smaller diameters than the anterior VL (Table 3).

\section{Dominant patterns}

Pertaining to the dominance patterns of the AV of the SCVS, the literature has reported the system being in haemodynamic balance ${ }^{4,7}$; furthermore, studies undertaken by Kawamata et al. and Tanriverdi et al. report variant patterns of dominance of these $A V^{8,20}$. The present study reports seven types of $A V$ dominance, viz. Equilibrium, dominant SSV, VL, VT, SSV/VL, SSV/VT as well as dominant VL/VT (Table 4, Fig. 2).

Dominant patterns of the $\mathrm{AV}$ were ascertained by the territorial drainage of the veins, and in some cases the absence of a vein played little to no role in the dominance of the AV. For example, in the absence of the VT, in some cases the territory of the VT was drained by the superior cortical veins and not the accompanying AV, i.e SSV and VL. The system was determined to be an SSV/VL dominant drainage pattern if the SSV and/or VL crossed into the territory of the VT.

The Equilibrium dominant pattern of drainage had the highest occurrence (54.5\%) in the present study and is aligned with the theory of haemodynamic balance of the SCVS, due to each AV draining their respective territories in a well-anastomosed system, as seen in Fig. 2A and 2B. However, the studies conducted by Kawamata et al. and Tanriverdi et al. report this type of dominant pattern of the AV in only $23.8 \%$ and $11.2 \%$ of the sample size, respectively ${ }^{9,20}$. Kawamata et al. had a predominance of the SSV/VT drainage pattern in $27.0 \%$ of the cohort studied, and Tanriverdi et al. reported a majority of the SSV/VL dominance pattern (19.5\%); however, the dominance patterns of SSV/VT and SSV/VL were reported as $3.0 \%$ and $2.5 \%$ in the present study, respectively ${ }^{4,20}$. Co-dominance of $\mathrm{VL}$ and $\mathrm{VT}$ was seen to have the second highest occurrence in the present study with $28.0 \%$ of the cohort presenting with the VL/VT dominant type; furthermore, this dominant type was determined to have a statistically significant relationship with sex ( $p=0.034)$ with males (36.2\%) having a higher presence of the VL/VT type than females (22.5\%) (Table 4, Fig. 2). Dominant patterns that presented with a statistically significant relationship with age were the VL $(p=0.037)$ and SSV/VL $(p=0.027)$ groups, the age group of 30-39 years had the highest occurrence of the respective dominant types (20.0\% VL dominant; $10.0 \%$ SSV/VL dominant) (Table 4). These dominant patterns of drainage are important in determining the safety of 
sacrifice of one of the AVs since this is largely based on collateral circulation and alternate drainage routes which would be available in well-anastomosed systems ${ }^{7}$.

Previous studies have hypothesised an inverse relationship between the dominant patterns of VL and VT based on laterality, stating that the presence of a VT dominance on the left hemisphere would lead to a VL dominance on the right hemisphere, and vice versa ${ }^{2,8,24,25}$. A Cramers $V$ correlation was carried out to determine the correlation of the types of dominant patterns of the AV, based on laterality (Table 5). The results of the present study indicate a weak correlation between the VL dominant pattern on the left hemisphere and VT dominance on the right (0.03), as well as a VL dominant AV on the right and a VT dominant $A V$ on the left hemisphere (0.04). Results from the Cramers $V$ correlation reported the highest correlation between dominant patterns of the AV, based on laterality, with a VL dominant AV on the left and right hemisphere having a medium correlation of 0.51 (Table 5).

\section{Conclusion}

Studies investigating the anatomy of cortical veins are imperative due to the course of these veins differing from cerebral arteries, as well as the high variability of these vessels. Since the AV are encountered during a common neurosurgical procedure, viz. pterional craniotomies, and due to the increased risk of iatrogenic injury to these AV, highlighting the anatomical variants regarding the presence, size and drainage patterns of these veins becomes important for neurosurgical perspectives. This study supports the statements of haemodynamic balance of the SCVS due to the seven types of dominant drainage patterns of the AV reported, as well as the high occurrence of the Equilibrium dominant pattern. The factor of laterality was not seen to be statistically significant amongst several results reported in this study, except for the diameter of the SSV; however, data reflects proportions of presence, diameter and dominant patterns based on laterality that must be noted by clinicians to aid in the planning of surgical corridors. Furthermore, the results from this study report occurrences of triple AV presence, which adds to the knowledge surrounding the AV.

\section{Declarations}

\section{Acknowledgements}

The authors wish to acknowledge the financial assistance of the National Research Foundation (NRF) towards this research (Grant number: 122254). The opinions expressed and conclusions arrived at are those of the authors and are not necessarily attributed to the NRF.

\section{Author Contributions}

J N: Project Development, Data collection, Data analysis, Manuscript writing and editing

R H: Project development, Data analysis, Manuscript editing

L L: Project development, Data analysis, Manuscript editing

\section{Conflicts of Interest}

The authors declare that they have no conflicts of interest to report.

\section{Ethical Approval}

Ethical clearance was obtained from the Biomedical Research Ethics Committee of the University of KwaZulu-Natal (UKZN) (BREC/00001676/2020).

\section{References}

1. Uddin, M. A., Ul Haq, T. \& Rafique, M. Z. Cerebral venous system anatomy.J Pak Med Assoc, 56(11):516-519(2006)

2. Lou, M. et al. Cerebral Venous System in Acute and Chronic Brain Injuries (Springer, 2019).

3. Stack, C. A. \& Cole, J. W. Cerebral Venous Thrombosis: A Clinical Overview, In: Ischemic Stroke of Brain, Intech Open Available at: http://dx.doi.org/10.5772/intechopen.79049 [Accessed: 09th September 2019] (2018)

4. Krings, T., Geibpraset, S., Cruz, J. P. \& terBrugge, K. G. Neurovascular anatomy in interventional radiology (Thieme Medical Publishers, 2015).

5. Winn, H. R. Youmans Neurological Surgery, Elsevier: Philadelphia, 6th Edition(2011)

6. Adachi, K., Hasegawa, M. \& Hirose, Y. Evaluation of venous drainage patterns for skull base meningioma surgery.Neurol Med Chir (Tokyo), 57:505512(2017)

7. Komiyama, M. Functional venous anatomy of the brain for neurosurgeons.Jpn J Neurosurg (Tokyo), 26:488-495(2017)

8. Tanriverdi, T., Al-Jehani, H., Poulin, N. \& Olivier, A. Superficial anastomotic veins: neurosurgical view depending on 251 craniotomies.Can J Neurol Sci, 36:65-71(2009)

9. Tubbs, R. S., Shoja, M. M. \& Loukas, M. Bergman's Comprehensive Encyclopaedia of Human Anatomic Variation (John Wiley \& Sons, 2016).

10. Bisaria, K. K. The superficial Sylvian vein in humans: with special reference to its termination. Anat Rec, 212:319-325(1985)

Page 9/11 
11. Appaji, A. C., Mohan, M., Kulkarni, R. \& Kulkarni, R. N. Anatomy of the vein of Labbe: a cadaveric study.Int J Anat Res, 5(1):3451-3456(2017)

12. Wangaryattawanich, P. et al. MR images of preoperative assessment of the bridging veins of the skull base.Radiographics, 36:244-257(2016)

13. Silva, S. P., Vilarinho, A., Carvalho, B. \& Vaz, R. Anatomical variations of the vein of Labbé: an angiographic study.Surg Radiol Anat(2014)

14. Rhoton, A. L. The cerebral veins. Neurosurgery, 51(1):159-205(2002)

15. Koperna, T., Tschabitscher, M. \& Khosp, E. The termination of the vein of "Labbe" and it's microsurgical significance.Acta Neurochir (Wien), 118:172175(1992)

16. Sindou, M., Auque, J. \& Jouanneau, E. Neurosurgery and the intracranial venous system. Acta Neurochir, 94:167-175(2005)

17. Grabner, G. et al. Post mortem validation of mri-identified veins on the surface of the cerebral cortex as potential landmarks for neurosurgery. Front. Neurosci, 11:355(2017)

18. Kilic, T. \& Akakin, A. Anatomy of cerebral veins and sinuses, Handbook on cerebral venous thrombosis. Front Neurol Neurosci. Basel, Karger(2008)

19. Bergsma, W. A bias-correction for Cramer's V and Tschuprow's T. JKSS, (3), https://doi.org/10.1016/j.jkss.2012.10.002 (2013). 42

20. Kawamata, T., Kiyoshi, M., Goto, N. \& Kohda, M. Morphometric anatomy of superficial cerebral veins and cerebral sulci.Showa Univ J Med Sci, 8(1):103111(1996)

21. Kazumata, K. et al. Operative anatomy and classification of the Sylvian veins for the transsylvian approach.Neurol Med Chir (Tokyo), 43:427-434(2003)

22. Suzuki, Y. \& Matsumoto, K. Variations of the superficial middle cerebral vein: classification using three-dimensional ct angiography. $A J N R$ Am $J$

Neuroradiol, 21:932-938(2000)

23. Avci, E., Dagtekin, A., Akture, E., Uluc, K. \& Baskaya, M. K. Microsurgical anatomy of vein of Labbe.Surg Radiol Anat, 33:569-573(2011)

24. Di Chiro, G. Angiographic patterns of cerebral convexity veins and superficial dural sinuses.Am J Roentgenol Radium Ther Nucl Med, 87:308-321(1962)

25. Lustig, L. R. \& Jackler, R. K. The vulnerability of the vein of Labbé during combined craniotomies of the middle and posterior fossae.Skull Base Surgery, $8(1)(1998)$

\section{Figures}

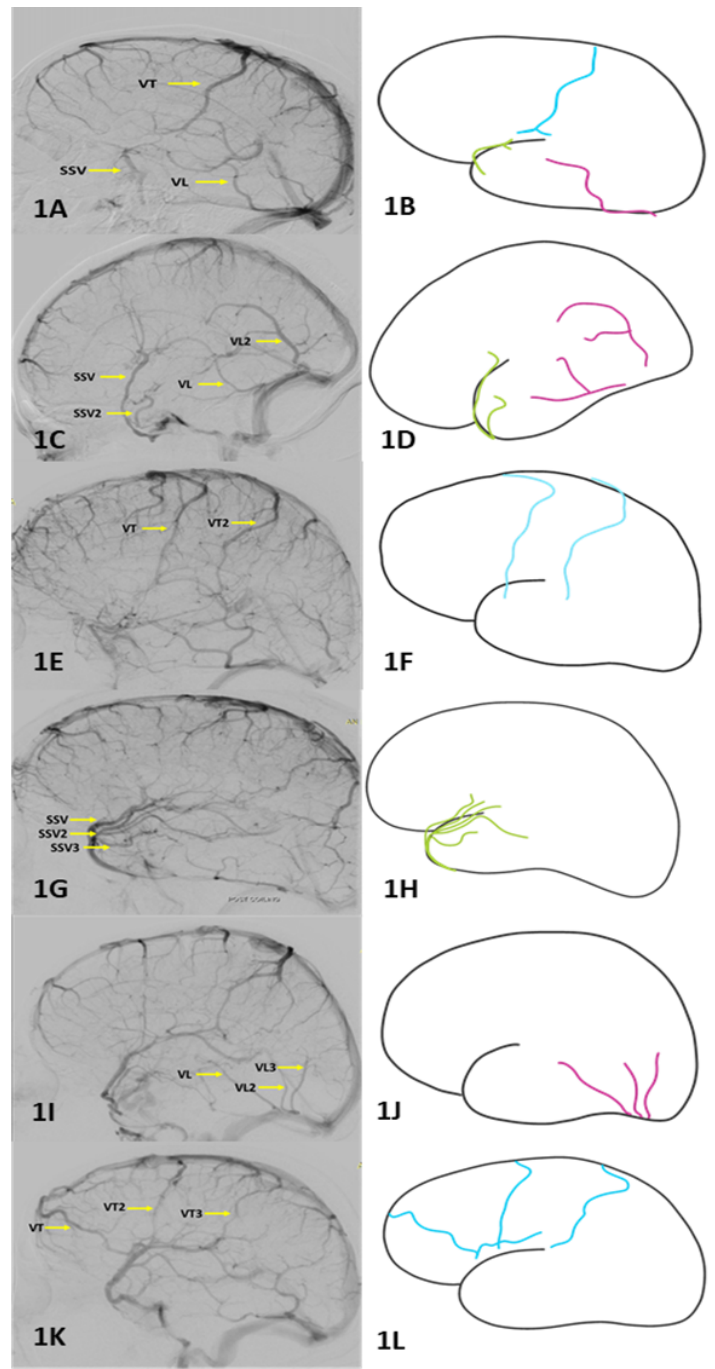

Figure 1 
Single, double and triple presence of the anastomotic veins in angiographic and schematic depictions A and B: Single presence of the AV C and D: Double SSV (SSV and SSV2) and Double VL (VL and VL2) E and F: Double VT (VT and VT2) G and H: Triple SSV (SSV, SSV2 and SSV3 - in ascending order, in an anteroposterior direction) I and J: Triple VL (VL, VL2 and VL3 - in ascending order, in an anteroposterior direction) K and L: Triple VT (VT, VT2 and VT3 - in ascending order, in an anteroposterior direction)

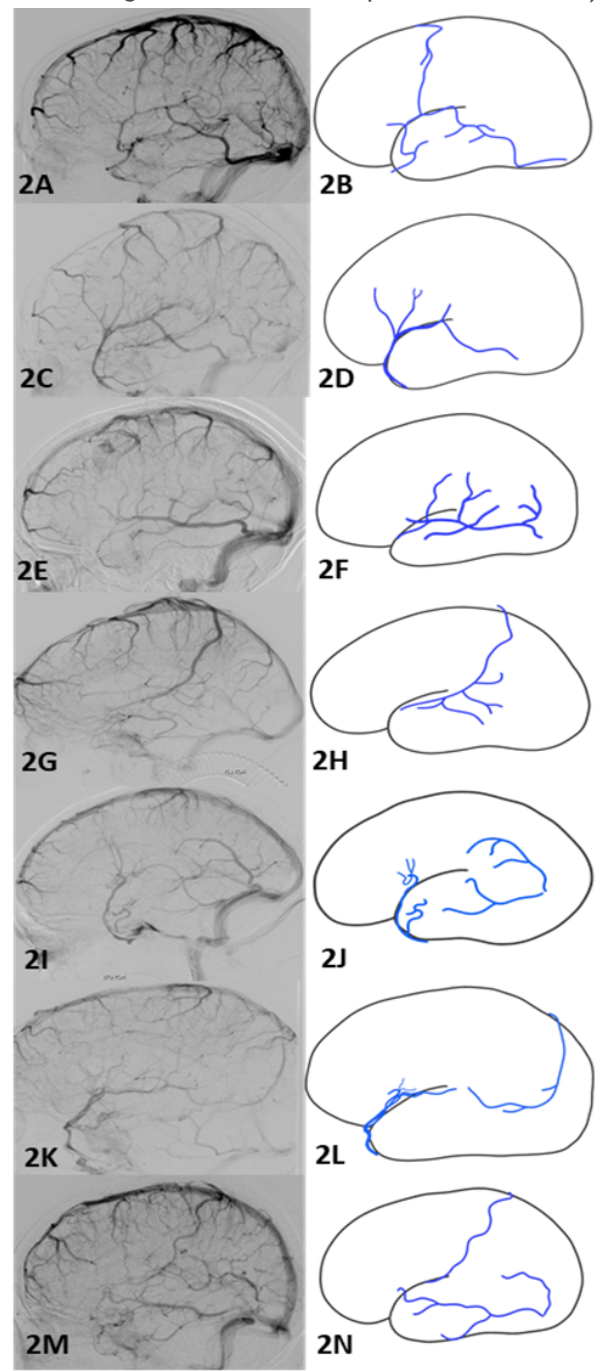

\section{Figure 2}

Angiographic images and schematic depictions of the dominant patterns of the anastomotic veins $2 \mathrm{~A}$ and $2 \mathrm{~B}$ represent an equilibrium or equidominant pattern $2 \mathrm{C}$ and $2 \mathrm{D}$ represent a singular dominant SSV pattern $2 \mathrm{E}$ and $2 \mathrm{~F}$ represent a singular VL dominant pattern $2 \mathrm{G}$ and $2 \mathrm{H}$ represent a singular $\mathrm{VT}$ dominant pattern $2 \mathrm{I}$ and $2 \mathrm{~J}$ represent a co-dominant SSV/VL pattern $2 \mathrm{~K}$ and $2 \mathrm{~L}$ represent a co-dominant SSV/VT pattern $2 \mathrm{M}$ and $2 \mathrm{~N}$ represent a codominant VL/VT pattern 